\title{
Stress Monitoring and Recent Advancements in Wearable Biosensors
}

\section{OPEN ACCESS}

Edited by:

Senentxu Lanceros-Mendez, Basque Center for Materials, Applications and Nanostructures,

Spain

Reviewed by:

Fatma Nese Kok,

Istanbul Technical University, Turkey

Costas Adam Charitidis,

National Technical University

of Athens, Greece

${ }^{*}$ Correspondence:

Cheyenne Samson

csamson2@binghamton.edu

Ahyeon Koh

akoh@binghamton.edu

Specialty section:

This article was submitted to Biomaterials,

a section of the journal Frontiers in Bioengineering and

Biotechnology

Received: 21 May 2020

Accepted: 11 August 2020

Published: 02 September 2020

Citation:

Samson C and Koh A (2020) Stress Monitoring and Recent

Advancements in Wearable

Biosensors.

Front. Bioeng. Biotechnol. 8:1037.

doi: 10.3389/fbioe.2020.01037

\section{Cheyenne Samson* and Ahyeon Koh*}

Department of Biomedical Engineering, Thomas J. Watson School of Engineering, Binghamton University, Binghamton, NY, United States

The stress response allows the body to overcome obstacles and prepare for threats, but sustained levels of stress can damage one's health. Stress has long been measured through physical tests and questionnaires that rely primarily on user-inputted data, which can be subjective and inaccurate. To quantify the amount of stress that the body is experiencing biologically, analytical detection of biomarkers associated with the stress response recently have been developed. Novel stress sensing devices focus on cortisol sweat sensing as a part of wearable, flexible devices. These devices promise a realtime, continuous collection of stress data that can be used in clinical diagnoses or for personal stress monitoring and mediation.

Keywords: stress biomarkers, stress sensors, wearable cortisol sensors, skin-interfaced cortisol sensors, stress analysis and evaluation

\section{INTRODUCTION}

Stress was first defined by in 1936 by Hans Selye, a pioneering endocrinologist from Hungary (Rochette and Vergely, 2017), as: "the non-specific response of the body to any demand." (Fink, 2009). Selye's subsequent stress experiments started the conversation about stress and its effect on the body (Rochette and Vergely, 2017). Selye's definition was quite narrow, placing a clear emphasis on only the biological aspects of stress. This has led to different definitions being used depending on context - behavioral scientists define stress as the perception of threat with resulting anxiety or discomfort (Fink, 2009), while neuroendocrinologists define it as any stimulus that triggers the secretion of the adrenocorticotropic hormone and glucocorticoids (Miller and O'Callaghan, 2002). In the context of this manuscript, stress is defined as any event that disrupts homeostasis, resulting in the release of hormones to return the body to homeostasis.

Stress is biologically associated with several disorders and related health problems. The industry standard for diagnosis of mental disorders (DSM-V) recognizes two stress-related disorders: Acute Stress Disorder (ASD) and Post Traumatic Stress Disorder (PTSD) (Fink, 2009; Bakhshian et al., 2013). Biologically, ASD and PTSD are associated with increased levels of cortisol and abnormal function of the hypothalamic-pituitary-adrenal (HPA) axis while being very different in their psychological severity (Bakhshian et al., 2013; Marin et al., 2019). The HPA axis works concurrently with the sympathoadrenal-medullary (SAM) axis of the sympathetic nervous system to stimulate the release of several hormones that prepare the body to survive a stressful situation. These axes are also instrumental in the appropriate termination of the release of stress hormones to maintain homeostasis and proper bodily function (Murison, 2016), and their effects can be visualized in Figure 1 below. In addition to PTSD and ASD, increased stress levels have been linked to decreased 
cardiovascular health (Poirat et al., 2018) and increased risk of anxiety-depressive symptoms (Marcatto et al., 2016).

The objective of this manuscript is to provide a comprehensive review of stress, what causes stress, and what methods and technologies are being used to measure stress.

\section{WHAT MAKES US STRESSED?}

Stress is experienced in a wide range of situations, including familial pressures, personal finances, academics, and more (Fairbrother and Warn, 2003; Reddy et al., 2018). Any stimulus that causes a stress response is a stressor (Schneiderman et al., 2005), which is defined as any environmental change that causes a shift toward a state of lower utility (Oken et al., 2015). In other words, a stressor is anything that causes a homeostatic imbalance and results in a biological or behavioral reaction to correct this imbalance (Oken et al., 2015; Murison, 2016). This imbalance, termed the stress response, varies in severity and duration from person to person (Oken et al., 2015). Homeostasis is the body's innate and dynamic ability to make physiological changes to maintain an adequate environment to perform all necessary physiological functions, and this definition has been the dominant explanation of self-regulation since it was coined by Walter Cannon in 1929 (Ramsay and Woods, 2014). Homeostasis, however, is a broad term, so the term allostasis is used to describe the physiological changes the body makes specifically in response to a stressor to maintain physiological balance (Schulkin and Sterling, 2019). According to allostatic principles, anticipation of a possible stressful event followed by appropriate regulation by the brain is the best way to physiologically regulate one's stress response (Ramsay and Woods, 2014). Therefore, the allostatic load that an individual experiences is highly variable, as the ability or inability to anticipate stressful events also varies based on the individual (Szalma, 2008; Schulkin and Sterling, 2019).

Evidence exists correlating neonatal experiences to the level of stress hormones released later in life (Champagne et al., 2003; Schneiderman et al., 2005). Champagne et al. (2003) showed that rats raised by nurturing mothers produced higher levels of serotonin, the happy hormone, later in their lives. It has been shown that serotonin is effective in suppressing the hormones related to panic, which is directly related to the stress response (Deakin and Graeff, 1991; Hood et al., 2006). Additionally, those rodent models raised by nurturing mothers, in turn, were great nurturers of their young (Champagne et al., 2003). In humans, childhood neglect has been shown to alter and shape the development of the HPA axis, the key controlling system of the stress reaction (Reilly and Gunnar, 2019).

While how each individual interprets stressors and reacts is highly variable, the biological response to stress is controlled by the HPA axis, which is responsible for the controlled release of hormones such as cortisol, ACTH, adrenaline, and noradrenaline (Murison, 2016). These hormones work together to give the body the best chance of survival against the perceived threat that a stressor poses (Oken et al., 2015). Indeed, stress-related disorders are psychological and caused by traumatic events but can be characterized biologically with this increased expression of cortisol within the body (Marin et al., 2019). Other factors that can influence HPA axis reactivity, and therefore the magnitude of the stress response, are age, sex, genetics, and prescription drugs (Zänkert et al., 2019).

\section{HOW TO MEASURE STRESS}

\section{Clinical Tests}

Several behavioral tests exist to quantify stress levels experienced by an individual (Oken et al., 2015). The Trier Social Stress Test (TSST) is commonly used to quantify acute stress by having the subject first perform a public speaking test followed by an arithmetic calculation. After these tasks are performed, analyses of the subject's saliva, blood, psychophysiological, and cognitive measures are made to assess stress levels (Allen et al., 2014). However, TSST is not perfect because of the great variability between room set-up, timing of events, etc. from trial to trial. This leads to inconsistencies and the inability to reproduce results using TSST (Labuschagne et al., 2019). Further efforts are being made to standardize TSST and other similar tests to make the results more reliable and reproducible (Dedovic et al., 2005; Labuschagne et al., 2019).

The Perceived Stress Scale (PSS) is a tool used by medical professionals to assess an individual's overall stress levels before any physical or psychological intervention (Nielsen et al., 2016). PSS is a simple survey that asks an individual several questions regarding the past month of their life on a scale from 0 (never) to 4 (very often). Examples of the questions that are asked are "How often have you been upset because of something that happened unexpectedly?" and "How often have you felt that things were going your way?" (Hewitt et al., 1992). These questions are meant to quantify the extent to which a subject has perceived their life as "unpredictable, uncontrollable, and overloading" in the past month (Nielsen et al., 2016), and therefore give insight into their stress level.

Structured similarly to PSS, the Kessler Psychological Distress Scale (K10) uses a set of 10 questions to quantify the level of mental distress a person is experiencing. These questions are answered based on the past month on a scale from 1 (none of the time) to 5 (all of the time) and include those such as "Did you feel nervous?" and "Did you feel depressed?" These questions were specifically curated to target depressive, anxious, and other psychologically disturbing feelings to assess the mental health levels of individuals. K10 has proven to be precise and reliable and allows for accurate determination of DSM-V cases and non-cases (Kessler et al., 2002).

\section{Biomarkers}

Stress can be quantified by measuring levels of different stress biomarkers that exist in bodily fluids. A biomarker is a molecule or other indicator that can give insight into the health of an individual via ex vivo analysis. Biomarkers provide quantifiable measures of biological processes, which gives medical professionals the ability to investigate issues 


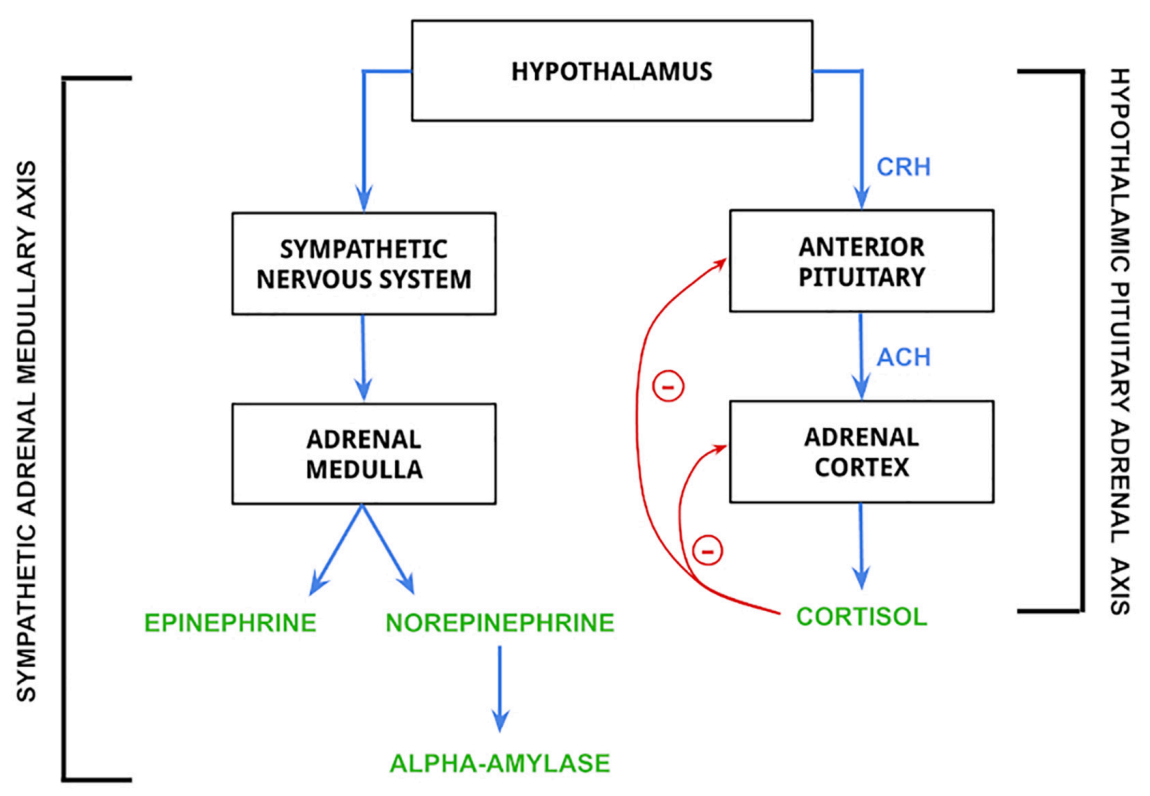

FIGURE 1 | Mechanism of release for epinephrine, norepinephrine, alpha-amylase, and cortisol. Biomarkers in the figure are controlled by the hypothalamus. The hypothalamic pituitary adrenal (HPA) axis is activated by hypothalamic release of corticotropin-releasing hormone $(\mathrm{CRH})$, which stimulates the secretion of adrenocorticotropin hormone $(\mathrm{ACH})$ from the anterior pituitary gland. $\mathrm{ACH}$ then acts on the adrenal cortex to stimulate the release of cortisol ( $\mathrm{Lanoix}$ and $\mathrm{Plusquellec}$, 2013; Antoun et al., 2017), which is regulated by negative feedback loops to both the anterior pituitary and adrenal cortex. The sympathetic adrenal medullary (SAM) axis is activated by the hypothalamus to stimulate a sympathetic nervous system response, which then stimulates the adrenal medulla to produce epinephrine and norepinephrine (Antoun et al., 2017). The release of norepinephrine is then responsible for the synthesis and release of alpha-amylase (Sawami et al., 2017).

happening within the body without having to perform a surgical procedure (Strimbu and Tavel, 2011). Non-invasive sampling of biofluids such as saliva and sweat provides the opportunity for continuous and real-time monitoring of analytes (Katchman et al., 2018), as well as providing a convenient and easy collection process for patient and collector. Some stress biomarkers, like cortisol, are present in more than one biofluid and cross-analysis can help validate results. Biomarkers are released and classified by two main systems in the body: hormones released by the endocrine system and neurotransmitters released by the nervous system (Steckl and Ray, 2018). The system that controls the release of the biomarker influences what biofluid it is present in. The main indicators of a stress response include dopamine, epinephrine, norepinephrine, serotonin, alpha-amylase, cortisol, and interleukin-6 (Goldstein, 2010; Ranabir and Reetu, 2011; Steckl and Ray, 2018; Dhama et al., 2019; Gug et al., 2019). Each one of these molecules plays a specific role in the stress response; all working to activate systems to ready the body to overcome the stressor evoking the response.

\section{Epinephrine and Norepinephrine}

Epinephrine and norepinephrine are hormones secreted by the adrenal gland that are vital in the evocation and regulation of the fight-or-flight response (Steckl and Ray, 2018). They cause increases in heart and respiratory rate, as well as suppress the immune system to shunt energy toward vital physiological systems to ready the body to respond and survive a perceived threat (Sanchez et al., 2003; Breed and Moore, 2012). Epinephrine and norepinephrine can be found in the blood at concentrations of $0-0.028$ and 0.06 $\mathrm{ng} / \mathrm{mL}$, respectively. They are also present in the urine at concentrations of $0-20$ and $15-80 \mathrm{ng} / \mathrm{mL}$, respectively (Steckl and Ray, 2018).

\section{Alpha-Amylase}

Alpha-amylase is an enzyme that cleaves large alpha-linked polysaccharides into glucose and maltose to be used as immediate energy sources. While alpha-amylase is not technically a biomarker, literature in recent years has shown that high levels of salivary alpha-amylase may indicate chronic stress (Nater and Rohleder, 2009; Vineetha et al., 2014; Steckl and Ray, 2018; Ali and Nater, 2020). The confirmation of salivary alpha-amylase as a reliable biomarker for stress will allow for greater variability in stress quantification approaches through saliva samples. Alphaamylase is present in the saliva at concentrations of 0.6$2.6 \mathrm{mg} / \mathrm{mL}$ (Nater and Rohleder, 2009). As seen in Figure 1, alpha-amylase release is controlled by the SAM axis, and the coactivation patterns between SAM and HPA axes is being studied as indications of a stress response (Wadsworth et al., 2020).

\section{Cortisol}

Cortisol is a glucocorticoid hormone, whose release is controlled by the central nervous system, more specifically the HPA axis. Cortisol is currently considered the gold standard for evaluating the activity of the HPA axis (Ali and Nater, 2020). Glucocorticoids are responsible for reallocation of energy to overcome real or anticipated stressors perceived during 
stress response (Herman et al., 2016). Cortisol synthesis and release are controlled by adrenocorticotropic hormone (ACTH), which is regulated by the levels of corticotropinreleasing hormone $(\mathrm{CRH})$ by the hypothalamus (Katsu and Iguchi, 2015). Cortisol fluctuates cyclically with the circadian rhythm, with concentrations peaking in the morning and decreasing throughout the 12 -h day (Rao and Androulakis, 2019). Cortisol binds to intracellular receptors to reduce inflammation, maintain blood pressure, suppress the immune system, and manage stress (Katsu and Iguchi, 2015). Cortisol is present in blood, saliva, sweat, urine, and cerebrospinal fluid. Especially, saliva and sweat are currently researched for stress devices because of their reliability and ease-ofcollection. The physiological ranges of cortisol in blood, saliva, and sweat are 30-230 ng/mL (Steckl and Ray, 2018), 0.1$10 \mathrm{ng} / \mathrm{mL}$ (Dhull et al., 2019), and 8-140 ng/mL (Jia et al., 2016), respectively.

\section{RECENT DEVELOPMENTS IN STRESS MONITORING SYSTEMS}

Through smartwatches (Hsiao and Chen, 2018), fitness trackers (Lee and Lee, 2018), and the general desire for smart, athome health services (Wiegard and Breitner, 2019), the general public is arguably more in-tune with their health and history than ever before (Kim and Kim, 2018). Many devices aimed at monitoring real-time stress rely on photoplethysmography data (Park et al., 2018), and some groups have integrated photoplethysmography data with other physiological signals such as heart rate variability (Mohan et al., 2016) or ECG data and respiratory signals (Chen et al., 2015). However, these signals are not the direct cause of a stress response, rather they are the physiological effect that results from the release of stress biomarkers in the body. As such, sensing of biomarkers may provide a more accurate way of stress sensing and will be the main focus of this manuscript. Several stress management applications are available for download on smartphones that provide coping strategies such as breathing, mindfulness, and mediation to help combat a stressful lifestyle. Apps which are evidence based are capable of supplementing medical care, but not at specifically quantifying stress levels (Coulon et al., 2016). To quantify stress more accurately, researchers are developing devices that can give relevant, concrete data through detection of specific stress biomarkers for stress monitoring. The recent developments in novel, non-invasive, wearable (or portable) sweat sensors will be discussed in further detail in next section.

\section{Salivary Sensors}

While salivary detection methods may not be conducive to wearable technologies, that does not mean that they have no place in point-of-care applications. A recent study by Liu et al. (2020) has shown that a portable salivary cortisol detection device can provide information that is just as useful as a wearable one. A portable differential pulse voltammetry (DVP) system was created that can be completely controlled via smartphone to deliver information to the individual about stress status. The working electrode, connected to a printed circuit board, is functionalized via anti-cortisol antibodies being linked to a gold nanoparticle electrode surface. An individual with this system can initiate a cortisol reading through the application, which then communicates to the DVP system to start a scan, which in turn relays the cortisol concentration back to the application via Bluetooth. This system has an acceptable linear range of $0.5-200 \mathrm{nM}$, or $0.18-$ $72.5 \mathrm{ng} / \mathrm{mL}$ (Liu et al., 2020). While the ability to have a wireless connection to a smartphone is extremely advantageous, this type of system does not allow for real-time analysis. Research into salivary alpha-amylase for stress assessment is also being done and a protype for handheld monitoring has been created (Hsiao et al., 2019). Given that salivary alphaamylase does not fluctuate with the circadian rhythm, as cortisol does, more emphasis on this area may be seen in the future. Nevertheless, the developments discussed further in this manuscript focus on cortisol, as its measurement is the most promising at this time.

\section{Sweat Cortisol Sensors}

Development and investigation of wearable sweat sensors has increased 10-fold in recent years. While the clinical relevance of biomarkers in sweat holds great potential, research is striving to determine the relevance for health monitoring (Chung et al., 2019). Devices being developed for cortisol analysis are validated with enzyme-linked immunosorbent assay (ELISA), an assay that uses antibodies coupled with an enzyme-mediated color change for colorimetric detection of an antigen. ELISA kits are widely available for purchase commercially for various antibodies and the biofluids they exist in (Gan and Patel, 2013).

Electrochemical detection methods are commonly used because of their ability to transduce biological signals into electrical ones through functionalized electrodes (Cho et al., 2020). One of the more common ways to functionalize an electrode is by taking advantage of the specificity of an antibody-antigen link, which uses the same theory behind ELISA detection. Figure $\mathbf{2}$ shows highlighted wearable devices for monitoring stress. A wearable, watch-like device "CortiWatch" has developed by Rice et al. (2019) that uses alpha-cortisol antibody-antigen detection as the functional sensor of chronoamperometric cortisol sensing unit. The working electrode was fabricated by immobilizing alphacortisol antibodies onto a gold electrode surface, which was circuited to a potentiostatic robust circuit board to collect data (Rice et al., 2019). While this device is wearable with all electronics housed in a 3D printed box, as shown in Figure 2A, it does not conform perfectly to the skin surface, which can leave gaps in sample collection and analysis. Another flexible wrist-worn device has been developed by Kinnamon et al. (2017) that overcomes this dilemma by integrating a flexible electrode system into their device. The electrode system contains molybdenum disulfide nanosheets functionalized with alpha-cortisol antibodies that allow for a dynamic sensing range of $1-500 \mathrm{ng} / \mathrm{mL}$. This system also shows the ability to perform real-time analysis of cortisol, given that it takes 

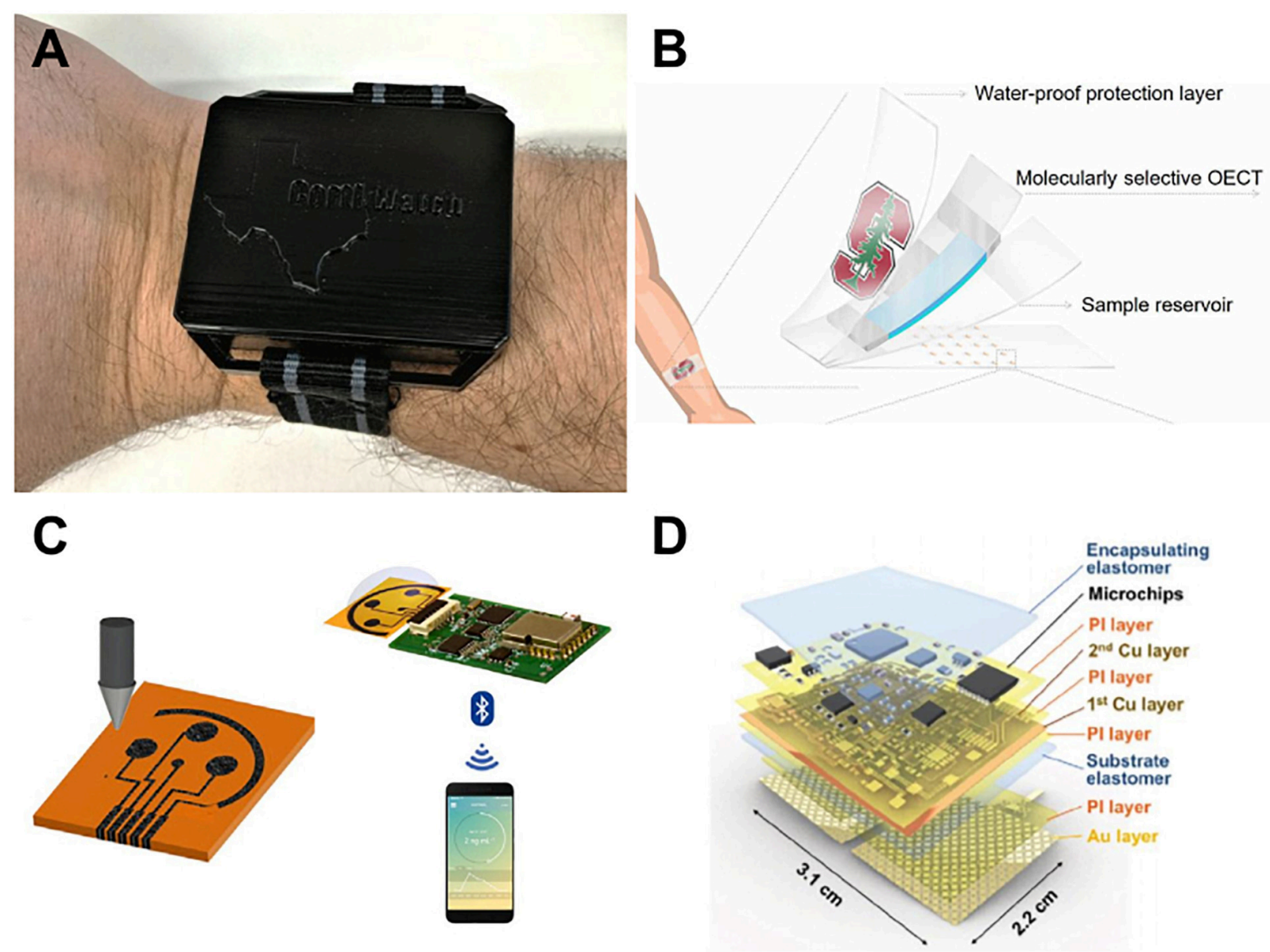

FIGURE 2 | Recent sweat stress sensor designs. (A) Wearable CortiWatch (Rice et al., 2019) reprinted via Creative Commons Attribution 4.0, (B) MIP sensor on flexible SEBS substrate (Parlak et al., 2018) reprinted via Creative Commons Attribution 4.0. (C) printed graphene-based wireless cortisol sensor, reprinted with permission from Torrente-Rodríguez et al., copyright 2020 by Elsevier (Torrente-Rodríguez et al., 2020). (D) galvanic skin response sensor SKINTRONICS (Kim et al., 2020) reprinted via Creative Commons Attribution 4.0.

only $3 \mathrm{~min}$ to perform a full frequency sweep for analysis (Kinnamon et al., 2017).

\section{Wearable Sensor Technologies for Stress Monitoring}

To move past rigid circuitry and electronics for wearable devices, many groups are opting to create soft, flexible sensors that can conform to the skin surface. A device developed by Lee et al. (2020) used cortisol-specific MX210 antibodies immobilized on a gold nanostructured surface to fabricate the working electrode in their design. The high density of antibodies that they were able to achieve optimized the sensitivity of their design and created a lower limit of detection of $1 \mathrm{pg} / \mathrm{mL}$ and dynamic range of $1 \mathrm{pg} / \mathrm{mL}-1 \mu \mathrm{g} / \mathrm{mL}$. This working electrode was a part of a flexible, wearable lab-on-a-patch platform developed with polydimethylsiloxane that used microfluidic sweat collection with detection via redox mediator reagent reaction and faradaic electrochemical impedimetric spectroscopy, as shown in Figure 2B; Lee et al. (2020). While using an antibody-antigen detection system is adequate, the sensor has the limitation of antibody-antigen instability and irreproducibility (Schonbrunn, 2014). To overcome this instability, an artificial molecularly imprinted polymer (MIP) has been developed by Parlak et al. (2018) The MIP was synthesized via copolymerization of a functional monomer with a cross-linker in the presence of cortisol - making a template for the cortisol binding sites. The cortisol was then eluted from the substrate, resulting in cortisol-specific binding sites across the MIP surface. Selectivity testing was performed in the presence of cortisol analogs including progesterone, cortisone, and testosterone to find that this novel MIP was adequately selective to measure cortisol concentration without significant interference from unwanted binding. The MIP was found to be reversible, reproducible, and reusable, effectively overcoming the shortcomings that antibodyantigen devices possess, while also being flexible and wearable due to development on a soft styrene-ethylene-butylene-styrene elastomer, as shown in Figure 2C; Parlak et al. (2018). Apart from cortisol sensing, Kim et al. (2020) reported a device they term "SKINTRONICS" which utilizes electrodermal sensing of 
galvanic skin response paired with temperature recording to determine stress levels. This flexible hybrid electronic device is skin-conformant, accurate, sensitive, and allows for the real-time capture of stress related data. As seen in Figure 2D, SKINTRONICS is a multilayered device with a wear time of up to $7 \mathrm{~h}$. The group was able to achieve galvanic skin response results comparable to those of commercially available devices with the same purpose, but with the added advantage of offering longterm, hands-free use (Kim et al., 2020). Other skin-interfaced sensing platforms are in development, indicating a trend toward flexible sensing (Sekar et al., 2019; Kim et al., 2020).

Bluetooth and other wireless data transfers are highly preferable in sensors that may eventually be used for personal use, as the typical person does not have access to complex computer systems that may be needed to visualize and interpret data. Torrente-Rodríguez et al. (2020) have proposed a device that seemingly contains all key features of a significant sweatsensing device that is flexible, wearable, accurate, and provides wireless data transmission to a smartphone via Bluetooth. The group has made use of a printed 3 graphene electrode system with an integrated Bluetooth module and microfluidic sample collection. The user is to initiate measurement via Bluetooth, which then triggers the electrode system through a potentiostat interface circuit and provides cortisol measurement in minutes, proving that this design may be applicable for realtime analysis (Torrente-Rodríguez et al., 2020). This design touts the ultimate all-in-one sensing platform which is totally wireless and integrated into a system that allows for an extremely userfriendly interface.

\section{CONCLUSION AND FUTURE DIRECTIONS}

Sweat sensing is at the forefront of wearable stress detection devices currently in development. These devices show great promise to quantify several sweat biomarkers, namely cortisol, to monitor the levels of stress that an individual is experiencing, as opposed to current stress mediating devices that rely on user input of data (Coulon et al., 2016). This biological information may be useful to supplement medical professional diagnoses of

\section{REFERENCES}

Ali, N., and Nater, U. M. (2020). Salivary Alpha-Amylase as a Biomarker of Stress in Behavioral Medicine. Int. J. Behav. Med. 27, 337-342. doi: 10.1007/s12529019-09843-x

Allen, A. P., Kennedy, P. J., Cryan, J. F., Dinan, T. G., and Clarke, G. (2014). Biological and Psychological Markers of Stress in Humans: Focus on the Trier Social Stress Test. Neurosci. Biobehav. Rev. 38, 94-124. doi: 10.1016/j.neubiorev. 2013.11.005

Antoun, M., Edwards, K. M., Sweeting, J., and Ding, D. (2017). The Acute Physiological Stress Response to Driving: A Systematic Review. PLoS One 12:1-13. doi: 10.1371/journal.pone.0185517

Bakhshian, F., Abolghasemi, A., and Narimani, M. (2013). Thought Control Strategies in the Patients with Acute Stress Disorder and PTSD. Proc. Soc. Behav. Sci. 84, 929-933. doi: 10.1016/j.sbspro.2013.06.676

Breed, M., and Moore, J. (2012). Endocrinology for Animal Behaviorists. Anim. Behav. 2016, 25-65. doi: 10.1016/B978-0-12-372581-3.00002-7 psychological disorders, as well as conditions such as Addison's and Cushing's diseases, which are characterized by low and high levels of basal cortisol, respectively (Ragnarsson, 2020; Saverino and Falorni, 2020). Future development of these devices may provide a direct user output instead of relying on additional components to visualize and analyze the data, similar to the attempts made by Liu et al. and Rodríguez et al. This may be achieved via the integration of near-field communication chips for remote data visualization via Bluetooth, which have been tested for flexibility (Jeong et al., 2017). Additionally, further research into other indicators of stress, such as alphaamylase, may see more attention as a measure of SAM axis activity (Hsiao et al., 2019). The multimodal wearable stress sensors will advance quality-of-life sensing systems that provide accurate, reliable, and viable physiological status enabling to apply artificial intelligences.

\section{AUTHOR CONTRIBUTIONS}

CS and AK conducted a literature review and wrote the manuscript. Both authors contributed to the article and approved the submitted version.

\section{FUNDING}

This research was supported by SUNY Binghamton University Start-Up funds. This material is based on research sponsored by Air Force Research Laboratory under agreement number FA8650-18-2-5402. The U.S. Government is authorized to reproduce and distribute reprints for government purposes notwithstanding any copyright notation thereon.

\section{ACKNOWLEDGMENTS}

We would like to express our appreciation for the following individuals for their expertise and advice throughout the writing of this manuscript: Dr. Lina Begdache, Paul Pearlmutter, and Nicholas Linehan.

Champagne, F. A., Francis, D. D., Mar, A., and Meaney, M. J. (2003). Variations in Maternal Care in the Rat as a Mediating Influence for the Effects of Environment on Development. Physiol. Behav. 79, 359-371. doi: 10.1016/ S0031-9384(03)00149-145

Chen, K., Fink, J., Roveda, R. D., Lane, D., Allen, J., and Vanuk, J. (2015). "Wearable Sensor Based Stress Management Using Integrated Respiratory and ECG Waveforms," in 2015 IEEE 12th Internation Conference on Wearable and Implantable Body Sensor Networks, (Cambridge, MA: IEEE), 1-6.

Cho, I., Kim, D. H., and Park, S. (2020). Electrochemical Biosensors: Perspective on Functional Nanomaterials for on-Site Analysis. Biomater. Res. 24, 1-12. doi: 10.1186/s40824-019-0181-y

Chung, M., Fortunato, G., and Radacsi, N. (2019). Wearable Flexible Sweat Sensors for Healthcare Monitoring: A Review. J. Royal Soc. Inter. 16:20190217. doi: 10.1098/rsif.2019.0217

Coulon, S. M., Monroe, C. M., and West, D. S. (2016). A Systematic, Multi-Domain Review of Mobile Smartphone Apps for Evidence-Based Stress Management. Am. J. Prevent. Med. 51, 95-105. doi: 10.1016/j.amepre.2016.01.026 
Deakin, J. F., and Graeff, F. G. (1991). 5-HT and Mechanisms of Defence. J. Psychopharmacol. 5, 305-315. doi: 10.1177/026988119100500414

Dedovic, K., Renwick, R., Mahani, N. K., Engert, V., Lupien, S. J., and Pruessner, J. C. (2005). The Montreal Imaging Stress Task: Using Functional Imaging to Investigate the Effects of Perceiving and Processing Psychosocial Stress in the Human Brain. J. Psychiatr. Neurosci. 30, 319-325.

Dhama, K., Latheef, S. K., Dadar, M., Samad, H. A., Munjal, A., Khandia, R., et al. (2019). Biomarkers in Stress Related Diseases/Disorders: Diagnostic, Prognostic, and Therapeutic Values. Front. Mol. Biosci. 6:91. doi: 10.3389/ fmolb.2019.00091

Dhull, N., Kaur, G., Gupta, V., and Tomar, M. (2019). Highly Sensitive and Non-Invasive Electrochemical Immunosensor for Salivary Cortisol Detection. Sensors Actuat. B Chem. 293, 281-288. doi: 10.1016/j.snb.2019.05.020

Fairbrother, K., and Warn, J. (2003). Workplace Dimensions, Stress and Job Satisfaction. J. Manager. Psychol. 18, 8-21. doi: 10.1108/02683940310459565

Fink, G. (2009). Stress: Concepts, Definition and History. Encycl. Neurosci. 2017, 549-555. doi: 10.1016/b978-0-12-809324-5.02208-2

Gan, S. D., and Patel, K. R. (2013). Enzyme Immunoassay and Enzyme-Linked Immunosorbent Assay. J. Investigat. Dermatol. 133, 1-3. doi: 10.1038/jid.2013. 287

Goldstein, D. S. (2010). Adrenal Responses to Stress. Cell. Mol. Neurobiol. 30, 1433-1440. doi: 10.1007/s10571-010-9606-9609

Gug, I. T., Tertis, M., Hosu, O., and Cristea, C. (2019). Salivary Biomarkers Detection: Analytical and Immunological Methods Overview. $\operatorname{Tr} A C$ 113, $301-$ 316. doi: 10.1016/j.trac.2019.02.020

Herman, J. P., Mcklveen, J. M., Ghosal, S., Kopp, B., Wulsin, A., Makinson, R., et al. (2016). Regulation of the Hypothalamic-Pituitary-Adrenocortical Stress Response. Comprehens. Physiol. 6, 603-621. doi: 10.1002/cphy.c150015. Regulation

Hewitt, P. L., Flett, G. L., and Mosher, S. W. (1992). The Perceived Stress Scale: Factor Structure and Relation to Depression Symptoms in a Psychiatric Sample. J. Psychopathol. Behav. Assess. 14, 247-257. doi: 10.1007/BF00962631

Hood, S. D., Hince, D. A., Robinson, H., Cirillo, M., Christmas, D., and Kaye, J. M. (2006). Serotonin Regulation of the Human Stress Response. Psychoneuroendocrinology 31, 1087-1097. doi: 10.1016/j.psyneuen.2006. 07.001

Hsiao, H., Chen, R. L. C., Chou, C., and Cheng, T. (2019). Hand-Held Colorimetry Sensor Platform for Determining Salivary $\alpha$-Amylase Activity and Its Applications for Stress Assessment. Sensors 19:1571. doi: 10.3390/s19071571

Hsiao, K. L., and Chen, C. C. (2018). What Drives Smartwatch Purchase Intention? Perspectives from Hardware, Software, Design, and Value. Telemat. Inform. 35, 103-113. doi: 10.1016/j.tele.2017.10.002

Jeong, J. H., Kim, J. H., and Oh, C. S. (2017). Quantitative Evaluation of Bending Reliability for a Flexible Near-Field Communication Tag. Microelectr. Reliab. 75, 121-126. doi: 10.1016/j.microrel.2017.06.030

Jia, M., Chew, W., Feinstein, T., Skeath, P., and Sternbery, E. (2016). Quantification of Cortisol in Human Eccrine Sweat by Liquid Chromatography - Tandem Mass Spectrometry. Analyst 141, 2053-2060. doi: 10.1016/j.physbeh.2017.03.040

Katchman, B. A., Zhu, M., Christen, J. B., and Anderson, K. S. (2018). Eccrine Sweat as a Biofluid for Profiling Immune Biomarkers. Proteo. Clin. Appl. 12, 1-8. doi: $10.1002 /$ prca.201800010

Katsu, Y., and Iguchi, T. (2015). Handbook of hormones: comparative endocrinology for basic and clinical research. Netherland: Elsevier Inc.

Kessler, R. C., Andrews, G., Colpe, L. J., Hiripi, E., Mroczek, D. K., Normand, S. L. T., et al. (2002). Short Screening Scales to Monitor Population Prevalences and Trends in Non-Specific Psychological Distress. Psychol. Med. 32, 959-976. doi: 10.1017/S0033291702006074

Kim, H., Kim, Y. S., Mahmood, M., Kwon, S., Zavanelli, N., Kim, H. S., et al. (2020). Fully Integrated, Stretchable, Wireless Skin-Conformal Bioelectronics for Continuous Stress Monitoring in Daily Life. Adv. Sci. 2000810, 1-10. doi: 10.1002/advs.202000810

Kim, S., and Kim, S. (2018). User Preference for an IoT Healthcare Application for Lifestyle Disease Management. Telecommun. Polic. 42, 304-314. doi: 10.1016/j. telpol.2017.03.006

Kinnamon, D., Ghanta, R., Lin, K. C., Muthukumar, S., and Prasad, S. (2017). Portable Biosensor for Monitoring Cortisol in Low-Volume Perspired Human Sweat. Scientific Rep. 7, 1-13. doi: 10.1038/s41598-017-13684-13687
Labuschagne, I., Grace, C., Rendell, P., Terrett, G., and Heinrichs, M. (2019). An Introductory Guide to Conducting the Trier Social Stress Test. Neurosci. Biobehav. Rev. 107, 686-695. doi: 10.1016/j.neubiorev.2019.09.032

Lanoix, D., and Plusquellec, P. (2013). Adverse Effects of Pollution on Mental Health: The Stress Hypothesis. OA Evidence Based Med. 48, 72-79. doi: 10. 13172/2053-2636-1-1-572

Lee, H. B., Meeseepong, M., Trung, T. Q., Kim, B. Y., and Lee, N. E. (2020). A Wearable Lab-on-a-Patch Platform with Stretchable Nanostructured Biosensor for Non-Invasive Immunodetection of Biomarker in Sweat. Biosensors Bioelectr. 156:112133. doi: $10.1016 /$ j.bios.2020.112133

Lee, S. Y., and Lee, K. (2018). Factors That Influence an Individual's Intention to Adopt a Wearable Healthcare Device: The Case of a Wearable Fitness Tracker. Technol. Forecast. Soci. Change 129, 154-163. doi: 10.1016/j.techfore.2018.01. 002

Liu, J., Xu, N., Men, H., Li, S., Lu, Y., Low, S. S., et al. (2020). Salivary Cortisol Determination on Smartphone-Based Di Ff Erential Pulse. Sensors 20:1422. doi: $10.3390 / \mathrm{s} 20051422$

Marcatto, F., Colautti, L., Larese, F., Luis, O., Di, L., Cavallero, C., et al. (2016). Work-Related Stress Risk Factors and Health Outcomes in Public Sector Employees. Safe. Sci. 89, 274-278. doi: 10.1016/j.ssci.2016.07.003

Marin, M. F., Geoffrion, S., Juster, R. P., Giguère, C. E., Marchand, A., Lupien, S. J., et al. (2019). High Cortisol Awakening Response in the Aftermath of Workplace Violence Exposure Moderates the Association between Acute Stress Disorder Symptoms and PTSD Symptoms. Psychoneuroendocrinology 104, 238-242. doi: 10.1016/j.psyneuen.2019.03.006

Miller, D. B., and O'Callaghan, J. P. (2002). Neuroendocrine Aspects of the Response to Stress. Metabolism 51, 5-10. doi: 10.1053/meta.2002.33184

Mohan, P. M., Nagarajan, V., and Das, S. R. (2016). "Stress Management from Wearable Photoplethysmographic Sensor Using Heart Rate Variability Data," in International Conference on Communication and Signal Processing, (Melmaruvathur), 1141-1144.

Murison, R. (2016). "The Neurobiology of Stress," in The Neuroscience of Pain, Stress, and Emotion: Psychological and Clinical Implications, eds M. Al'Absi and M. A. Flaten (Massachusetts, CA: Academic Press), 29-49. doi: 10.1016/C20130-16065-5

Nater, U. M., and Rohleder, N. (2009). Salivary Alpha-Amylase as a Non-Invasive Biomarker for the Sympathetic Nervous System: Current State of Research. Psychoneuroendocrinology 34, 486-496. doi: 10.1016/j.psyneuen.2009.01.014

Nielsen, M. G., Ørnbøl, E., Vestergaard, M., Bech, P., Larsen, F. B., Lasgaard, M., et al. (2016). The Construct Validity of the Perceived Stress Scale. J. Psychosomat. Res. 84, 22-30. doi: 10.1016/j.jpsychores.2016.03.009

Oken, B. S., Chamine, I., and Wakeland, W. (2015). A Systems Approach to Stress, Stressors and Resilience in Humans. Behav. Brain Res. 282, 144-154. doi: 10.1016/j.bbr.2014.12.047

Park, J., Kim, J., and Kim, S. (2018). "Prediction of Daily Mental Stress Levels Using a Wearable Photoplethysmography Sensor," in TENCON 2018 - 2018 IEEE Region 10 Conference, (New Jersey, NJ: IEEE), 1899-1902.

Parlak, O., Keene, S. T., Marais, A., Curto, V. F., and Salleo, A. (2018). Molecularly Selective Nanoporous Membrane-Based Wearable Organic Electrochemical Device for Noninvasive Cortisol Sensing. Sci. Adv. 4:eaar2904. doi: 10.1126/ sciadv.aar2904

Poirat, L., Gaye, B., Perier, M. C., Thomas, F., Guibout, C., Climie, R. E., et al. (2018). Perceived Stress Is Inversely Related to Ideal Cardiovascular Health: The Paris Prospective Study III. Int. J. Cardiol. 270, 312-318. doi: 10.1016/j.ijcard. 2018.06.040

Ragnarsson, O. (2020). Cushing's Syndrome - Disease Monitoring: Recurrence, Surveillance with Biomarkers or Imaging Studies. Best Pract. Res. Clinic. Endocrinol. Metabol. 34:101382. doi: 10.1016/j.beem.2020.101382

Ramsay, D. S., and Woods, S. C. (2014). Clarifying the Roles of Homeostasis and Allostasis in Physiological Regulation. Psychol. Rev. 121, 225-247. doi: 10.1037/ a0035942

Ranabir, S., and Reetu, K. (2011). Stress and Hormones. Indian J. Endocrinol. Metabol. 15, 18-22. doi: 10.4103/2230-8210.77573

Rao, R., and Androulakis, I. P. (2019). Hormones and Behavior The Physiological Significance of the Circadian Dynamics of the HPA Axis: Interplay between Circadian Rhythms. Allostasis and Stress Resilience. Hormones Behav 110, 77-89. doi: 10.1016/j.yhbeh.2019.02.018 
Reddy, K. J., Menon, K. R., and Thattil, A. (2018). Academic Stress and Its Sources among University Students. Biomed. Pharmacol. J. 11, 531-537. doi: 10.13005/ $\mathrm{bpj} / 1404$

Reilly, E. B., and Gunnar, M. R. (2019). Neglect, HPA Axis Reactivity, and Development. Int. J. Dev. Neurosci. 78, 100-108. doi: 10.1016/j.ijdevneu.2019. 07.010

Rice, P., Upasham, S., Jagannath, B., Manuel, R., Pali, M., and Prasad, S. (2019). CortiWatch: Watch-Based Cortisol Tracker. Future Sci. OA 5:FSO416. doi: 10.2144/fsoa-2019-2061

Rochette, L., and Vergely, C. (2017). Hans Selye and the Stress Response: 80 Years after His 'Letter' to the Editor of Nature. Annal. de Cardiol. et d'Angeiol. 66, 181-183. doi: 10.1016/j.ancard.2017.04.017

Sanchez, A., Menezes, M. L., and Pereira, O. C. M. (2003). Changes in Norepinephrine and Epinephrine Concentrations in Adrenal Gland of the Rats Submitted to Acute Immobilization Stress. Pharmacol. Res. 48, 607-613. doi: 10.1016/S1043-6618(03)00241-X

Saverino, S., and Falorni, A. (2020). Autoimmune Addison's Disease. Best Pract. Res.: Clinic. Endocrinol. Metabol. 34:101379. doi: 10.1016/j.beem.2020.101379

Sawami, K., Nakagawa, H., Katahata, Y., and Suishu, C. (2017). Verification of Preventive Effect of Dual-Task and N-Back Task- Incorporated Music Therapy against Dementia. Neurochem. Neuropharmacol. 14: 1381-1393. doi: 10.4172/ 2469-9780.1000115

Schneiderman, N., Ironson, G., and Siegel, S. D. (2005). Stress and Health: Psychological, Behavioral, and Biological Determinants. Ann. Rev. Clinic. Psychol 1, 607-628. doi: 10.1146/annurev.clinpsy.1.102803.144141

Schonbrunn, A. (2014). Editorial: Antibody Can Get It Right: Confronting Problems of Antibody Specificity and Irreproducibility. Mole. Endocrinol. 28, 1403-1407. doi: 10.1210/me.2014-1230

Schulkin, J., and Sterling, P. (2019). Allostasis: A Brain-Centered, Predictive Mode of Physiological Regulation. Trends Neurosci. 42, 740-752. doi: 10.1016/j.tins. 2019.07.010

Sekar, M., Pandiaraj, M., Bhansali, S., Ponpandian, N., and Viswanathan, C. (2019). Carbon Fiber Based Electrochemical Sensor for Sweat Cortisol Measurement. Scientific Rep. 9, 1-14. doi: 10.1038/s41598-018-37243-w

Steckl, A. J., and Ray, P. (2018). Stress Biomarkers in Biological Fluids and Their Point-of-Use Detection. ACS Sensors 3, 2025-2044. doi: 10.1021/acssensors. $8 \mathrm{~b} 00726$

Strimbu, K., and Tavel, J. A. (2011). What Are Biomarkers? Curr. Opin. HIV AIDS 5, 463-466. doi: 10.1097/COH.0b013e32833ed177. What
Szalma, J. L. (2008). "Individual Differences in Stress Reaction," in Performance Under Stress, eds P. A. Hancock and J. L. Szalma (England: Hampshire), 323-357.

Torrente-Rodríguez, R. M., Tu, J., Yang, Y., Min, J., Wang, M., Song, Y., et al. (2020). Investigation of Cortisol Dynamics in Human Sweat Using a GrapheneBased Wireless MHealth System. Matter 2, 921-937. doi: 10.1016/j.matt.2020. 01.021

Vineetha, R., Pai, K. M., Vengal, M., Gopalakrishna, K., and Narayanakurup, D. (2014). Usefulness of Salivary Alpha Amylase as a Biomarker of Chronic Stress and Stress Related Oral Mucosal Changes - a Pilot Study. J. Clin. Exp. Dentistr. 6, e132-e137. doi: 10.4317/jced.51355

Wadsworth, M. E., Bendezu, J. J., and Mcdonald, A. (2020). Co-Activation of SAM and HPA Responses to Acute Stress: A Review of the Literature and Test of Differential Associations with Preadolescents '. Int. External. Dev. Psychobiol. 61, 1079-1093. doi: 10.1002/dev.21866

Wiegard, R. B., and Breitner, M. H. (2019). Smart Services in Healthcare: A Risk-Benefit-Analysis of Pay-as-You-Live Services from Customer Perspective in Germany. Electr. Market. 29, 107-123. doi: 10.1007/s12525-017-02 $74-271$

Zänkert, S., Bellingrath, S., Wüst, S., and Kudielka, B. M. (2019). HPA Axis Responses to Psychological Challenge Linking Stress and Disease: What Do We Know on Sources of Intra- and Interindividual Variability? Psychoneuroendocrinology 105, 86-97. doi: 10.1016/j.psyneuen.2018.10.027

Disclaimer: The views and conclusions contained herein are those of the authors and should not be interpreted as necessarily representing the official policies or endorsements, either expressed or implied, of Air Force Research Laboratory, the U.S. Government, or SEMI-FlexTech.

Conflict of Interest: The authors declare that the research was conducted in the absence of any commercial or financial relationships that could be construed as a potential conflict of interest.

Copyright (c) 2020 Samson and Koh. This is an open-access article distributed under the terms of the Creative Commons Attribution License (CC BY). The use, distribution or reproduction in other forums is permitted, provided the original author(s) and the copyright owner(s) are credited and that the original publication in this journal is cited, in accordance with accepted academic practice. No use, distribution or reproduction is permitted which does not comply with these terms. 